\title{
A DSP based Controller Design for Synchronous Buck Converter
}

\author{
Shashi Kumar.M \\ M'Tech, Dept of EEE \\ Reva Institute of Technology,Bengaluru, Karnataka, India \\ Siddaramaiah.K.S \\ M'Tech, Dept of EEE \\ Reva Institute of Technology,Bengaluru, Karnataka, India \\ Prof.Vishwanath.V \\ Asst Prof, Dept of EEE \\ Reva Univertsity,Bengaluru, Karnataka, India
}

\begin{abstract}
Based on the high performance digital signal processing (DSP), analog digital converter (ADC), and pulse width modulator (PWM), a general digital controller based on DSP is designed for synchronous buck converter. Based on main circuit design, basic principle of PWM based DSP controller i.e.; TMS320F28335 is discussed and studied in detail. A universal controller is designed by analysis of PWM generation by DSP. A prototype is developed and constructed based on PWM by DSP for the control of synchronous buck converter. The static tests are performed which tests that the output voltage changes little by input voltage or load change of synchronous buck converter. The experiments and tests shows that the designed system has high efficiency, high accuracy, fast dynamic response and robustness.
\end{abstract}

Keywords- PWM, Synchronous buck converter, DSP Controller

\section{I.INTRODUCTION}

Synchronous buck converter has been used widely in many areas and in our everyday life like communications, IT, aerospace etc. because of its high efficiency, small size and structure and so on [1]. Buck converter has become the focus in recent years for its demand for high performance [2]. The application of sliding mode control theory and technology in buck converter has achieved good results [3]-[5]. But this method theoretically requires infinite fast switching speed of device. For this reason, many sliding mode control system uses hysteresis technique to limit the switching device frequency. But its very difficult to determine the width of hysteresis band because of its uncertainties in switching frequency, which is difficult for input and output filtering. In few sliding mode control, there will be a fixed frequency [6]. The output voltage of the converter is controlled by sliding mode controller by varying the PWM signal duty cycle. This solves the issue of switching devices of sliding mode controller affected by speed. The above method lack theoretical basis, control accuracy is low, slow dynamic response. Now a day's advancement in high speed DSP with low price and its high performance [7], buck converter can be controlled by DSP digitally. For getting point load power in DC, dynamic response capacity is very important factor. Normally the converters which are controlled digitally will have high switching frequency, which gives high performance. In this paper a synchronous buck converter is taken, which uses DSP chip to design controller. When there is any fast change I input voltage or load, output fluctuations will be low and it recovers rapidly because of the minimum recovery response time.

\section{STUDY OF SYNCHRONOUS BUCK CONVERETER}

The figure 1 shows the synchronous buck converter where vi is input voltage and vo is output voltage. The major difference between the normal buck converter and synchronous buck converter is that the diode in buck converter is replaced by a mosfet in synchronous buck converter. This second mosfet in place of diode will have low voltage drop compared to diode, this increases circuit efficiency. This is very important in high current low voltage applications. The mosfets ON and OFF is determined by PWM signal. Both mosfets must not be ON at 
the same time. When s1 is ON s2 must be turned off. When s2 is ON s1 must be off. Some time a dead time is given to avoid short circuit.

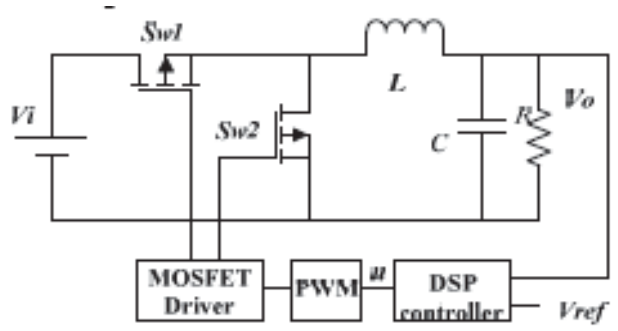

Fig. 1 Synchronous Buck Converter

III. ANALYSIS OF SYNCHRONOUS BUCK CONVERETR

MODE 1: When switch $\mathrm{S} 1$ is closed, $\mathrm{S} 2$ is open and the inductor gets charged and the equivalent circuit is shown in figure 2.

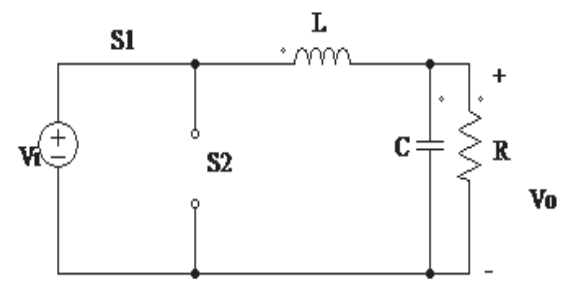

FIg. 2 when S1 is close

The voltage across inductor is given by

$$
v_{L}=V_{s}-V_{o}=L \frac{d i_{L}}{d t}
$$

Change in current is given by

$$
\left(\Delta i_{L}\right)_{\text {closed }}=\left(\frac{V_{s}-V_{o}}{L}\right) D T
$$

MODE 2: When switch S2 is closed, $\mathrm{S} 1$ is open and the inductor gets discharged and the equivalent circuit is shown in figure 3.

The voltage across inductor is given by

$$
v_{L}=-V_{o}=L \frac{d i_{L}}{d t}
$$

Change in current is given by

$$
\left(\Delta i_{L}\right)_{\mathrm{open}}=-\left(\frac{V_{o}}{L}\right)(1-D) T
$$

For steady state operation, net change in inductor current must be zero 


$$
\left(\Delta i_{L}\right)_{\text {closed }}+\left(\Delta i_{L}\right)_{\text {open }}=0
$$

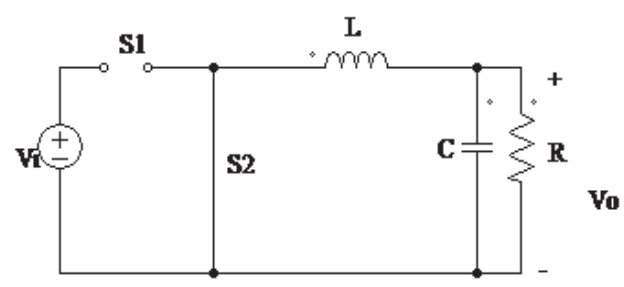

Fig. 3 when S2 is closed

Substituting equation (2) and (4) in equation (5)

$$
\left(\frac{V_{s}-V_{o}}{L}\right)(D T)-\left(\frac{V_{o}}{L}\right)(1-D) T=0
$$

Solving equation (6) for vo,

$$
V_{o}=V_{s} D
$$

Inductor current is same as current in load resistor and average capacitor current must be zero for steady state operatioN

$$
I_{L}=I_{R}=\frac{V_{o}}{R}
$$

Lmin is the minimum inductance required,

$$
L_{\text {min }}=\frac{(1-D) R}{2 f}
$$

Let the inductor be 25 percent larger than the minimum to ensure that inductor current is continuous

$$
L=1.25 L_{\min }
$$

The capacitor equation is given by

$$
C=\frac{1-D}{8 L\left(\Delta V_{o} / V_{o}\right) f^{2}}
$$

\section{TMS320F28335 DSP ARCHITECTURE}

The TMS320F28335 Digital Signal Controller (DSC) is capable of executing six basic operations in a single instruction cycle. The TMS320F28335 block diagram is shown in figure 4 . It can be divided into many functional units:Internal and external Bus System, Central Processing Unit (CPU), Internal Memory Sections, Control Peripherals, Communication Channels, Direct Memory Access Controller (DMA), Interrupt Management Unit (PIE) and Core Time Unit, Real - Time Emulation Interface 


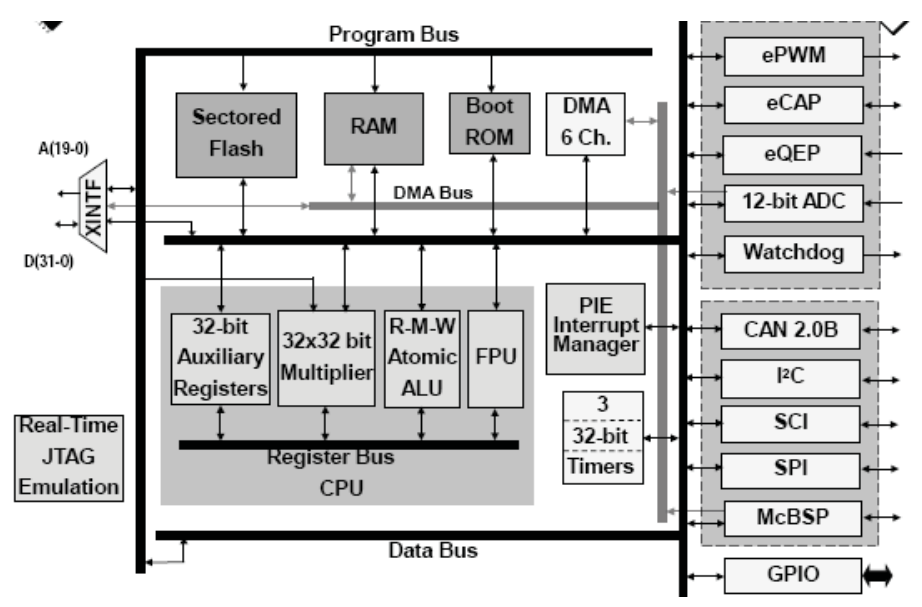

Fig. 4 TMS320F28335 Block diagram

\section{V . DIGITAL INPUT OUTPUT UNIT}

All digital I/O's are grouped together into "Ports", called GPIO-A, B and C. Here GPIO means "general purpose input output". The F28335 features a total of 88 I/O-pins, called GPIO 0 to GPIO 87. The device comes with so many additional internal units, that not all features could be connected to dedicated pins of the device package at any one time. The solution is multiplex. This means, one single physical pin of the device can be used for up to 4 different functions and it is up to the programmer to decide which function is selected. The block diagram of one physical pin of the device is shown below in figure 5.The term "Input Qualification" refers to an additional option for digital input signals at GPIO0-63. When this feature is used, an input pulse must be longer than the specified number of clock cycles to be recognized as a valid input signal. This is useful for removing input noise. Register Group "GPxPUD" can be used to disable internal pull-up resistors to leave the voltage level floating or high impedance. When a digital I/O function is selected, then register group GPxDIR defines the direction of the Input or Output. Clearing a bit position to zero configures the line as an input, setting the bit position to 1 configures the line as an output. A data read from an input line is performed with a set of GPxDAT registers. A data write to an output line can also be performed with registers GPXDAT. Additionally, there are 3 more groups of registers:GPxSET, GPxCLEAR, GPxTOGGLE

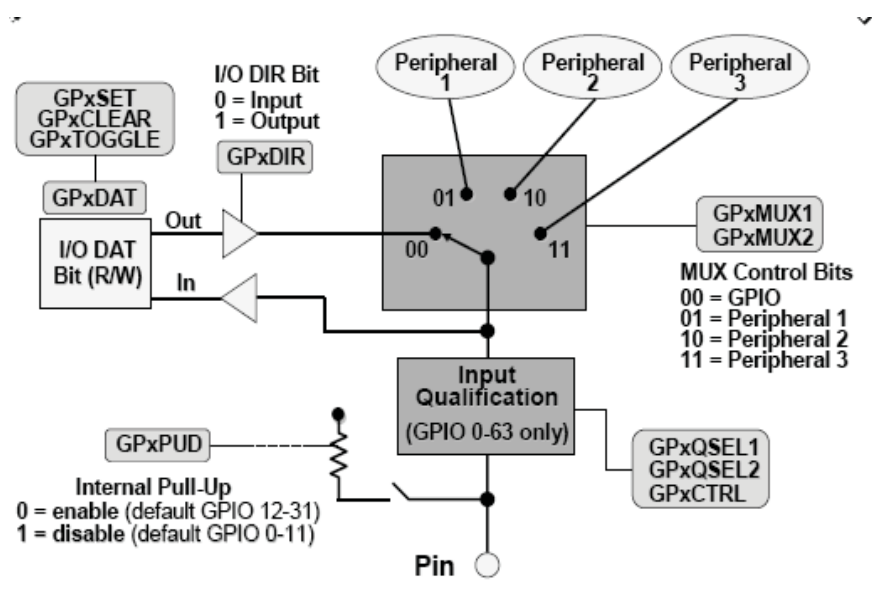

Fig. 5 Block diagram of TMS230F28335 GPIO Pin 


\section{ENHANCED PULSE WIDTH MODULATION}

Enhanced Pulse Width Modulation (ePWM) unit is controlled by its own logic block, as shown in figure 6. This logic is able to automatically generate signals on different time events and also to request various interrupt services from the F28335 PIE interrupt system, to support its operational modes. Main feature of an ePWM is its ability to start the Analogue to Digital Converter (ADC) without software interaction, directly from an internal hardware event. There are two basic operating modes of the ePWM system: (1) standard ePWM 16-bit mode and (2) 24-bit High Resolution PWM mode (HRPWM). The purpose of an ePWM unit is to generate a single ended signal or a pair of output signals, called EPWMxA and EPWMxB, which are related to each other. The lower case letter $\mathrm{x}$ is a placeholder for the number of the ePWM unit.

\section{PWM GENERATION}

The load voltage and current are sensed. The sensed output voltage is compared with a reference voltage of $12 \mathrm{~V}$ by using an error amplifier. The output of error amplifier is given to a comparator. This comparator is also fed with a current sensor output. The comparator output is fed to DSP TMS320F28335 which has built in Analog to Digital Converter (ADC). The PWM signal generated from DSP depends on comparator output value. If the comparator output is more, then this corresponds to more load voltage accordingly DSP will produce a PWM signal of less width. If the comparator output is less then this corresponds to less load voltage accordingly DSP will produce a PWM signal of more width. The DSP TMS320F28335 has the following PWM modules: ePWM Time-Base Module, ePWM Compare Module, ePWM Action Qualifier Module, ePWM Dead-Band Module, ePWM PWM Chopper Module, ePWM Trip-Zone Module and ePWM Event-Trigger Module. In the proposed study, ePWM compare module is used and PWM signals are generated. The DSP is programmed to generate PWM signals. The different source and header files are developed to sense the ADC output available in order to generate PWM signals.

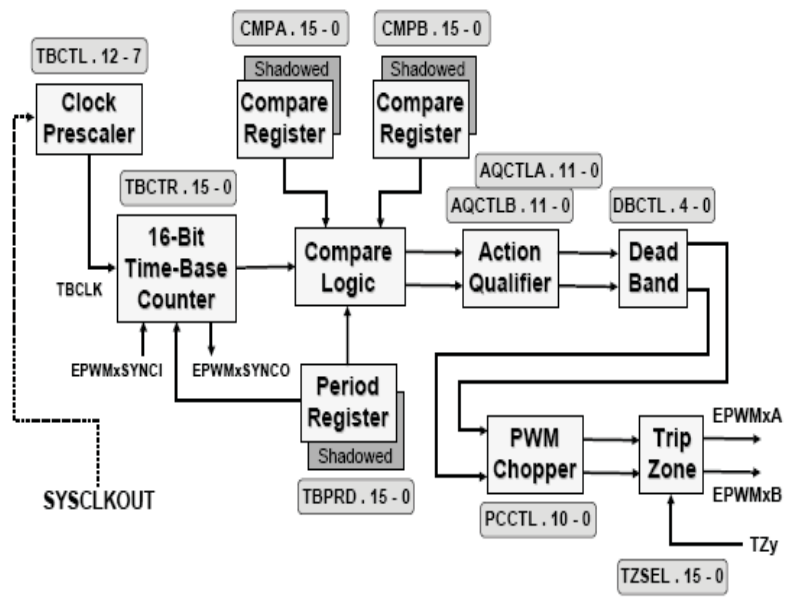

Fig. 6 Block diagram of ePWM

\section{PROGRAM DESIGN}

DSP TMS320F28335 chip is used as main control core. Main language of software is C program and assembly language written using the CCS 5.0 tool. Program flow chart is shown in figure 7.

\section{SIMULATION RESULTS}

The synchronous buck converter is simulated using MATLAB Simulink and simulink diagram and waveforms are shown in Fig.8. 

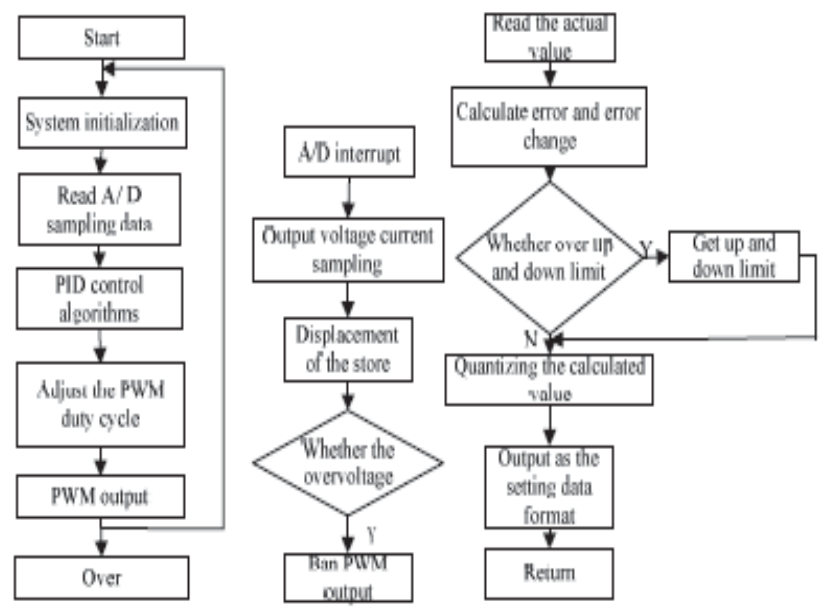

Main progeran fundaut

Interupt program flowchart PID control algorithm flowchart

Fig. 7 Flow chart of program

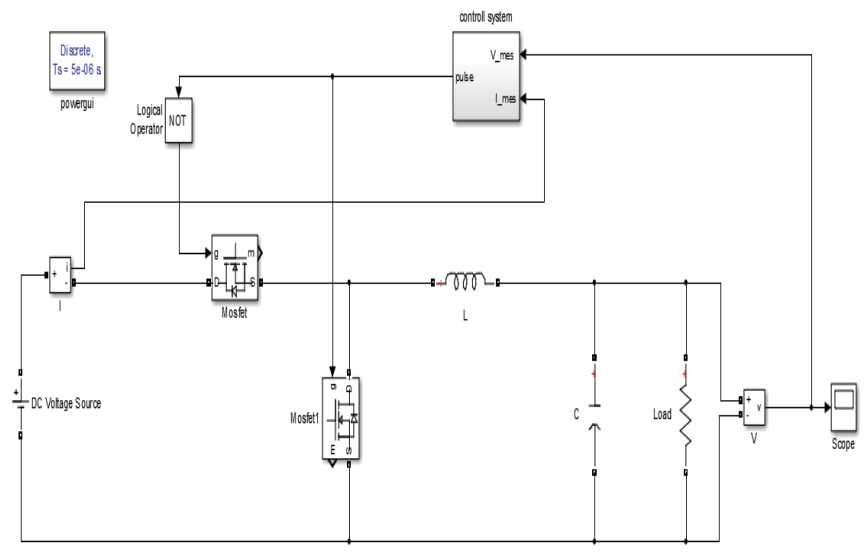

Fig. 8 MATLAB-Simulink Diagram of Synchronous buck converter

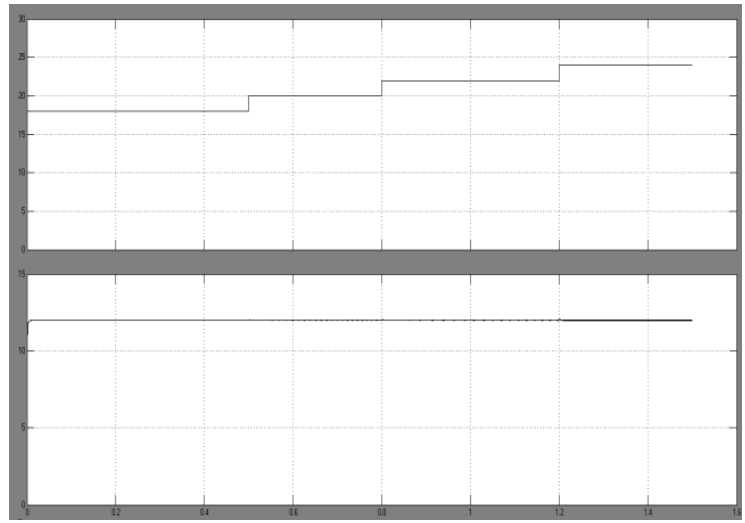

Fig. 9 MATLAB-Simulink Line regulation wave forms of Synchronous Buck converter 


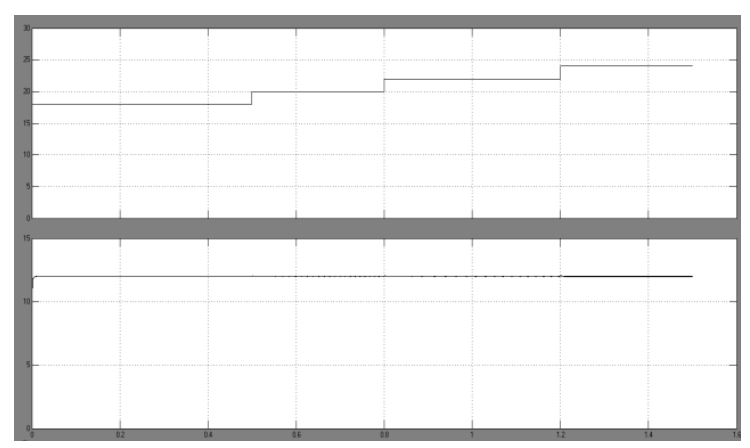

Fig. 10 MATLAB-Simulink Load regulation wave forms of Synchronous Buck converter

\section{EXPERIMENTAL RESULTS AND ANALYSIS}

The Synchronous buck converter is designed simulated in MATLAB Simulink and then prototype is developed using DSP. The converter is designed for $50 \%$ duty cycle. In designed prototype $12 \mathrm{v}$ battery is taken as input source and the voltage is regulated at $6 \mathrm{v}$. In proposed work, DSP TMS320F28335 is interfaced to synchronous buck converter. The below picture show the prototype interfaced with DSP.

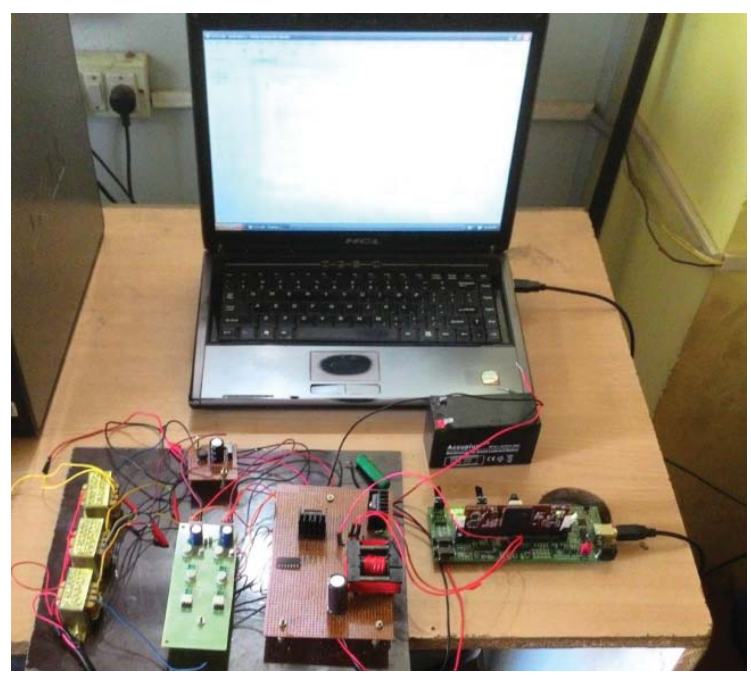

Fig 11. Prototype of synchronous buck converter

The pwm waveforms of synchronous buck convereter is generated using DSP and is captured using DSO and is show below in fig 12.The output voltage waveforms of synchronous buck convereter is generated using DSP and is captured using DSO and is show in fig 13

\section{CONCLUSSION}

The DSP based controller for synchronous buck converter is studied and designed using PWM technique. Detailed study is done on DSP and PWM, and experimental prototype is developed. The line and load regulation tests are done and tabulated. The DSP based controller have good robustness, high control accuracy and fast dynamic response when the system's internal parameters changed. 


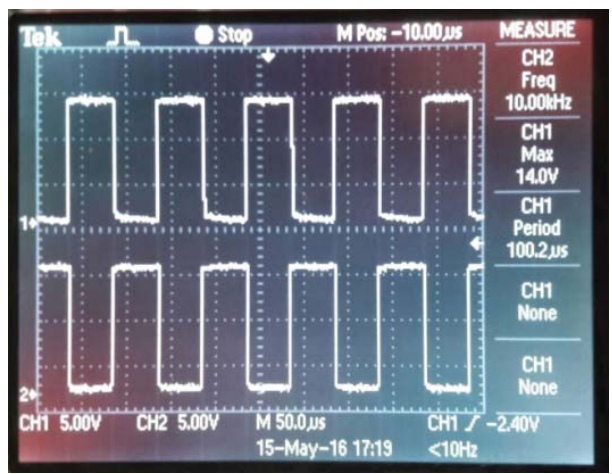

Fig 12. PWM waveforms of synchronous buck converter

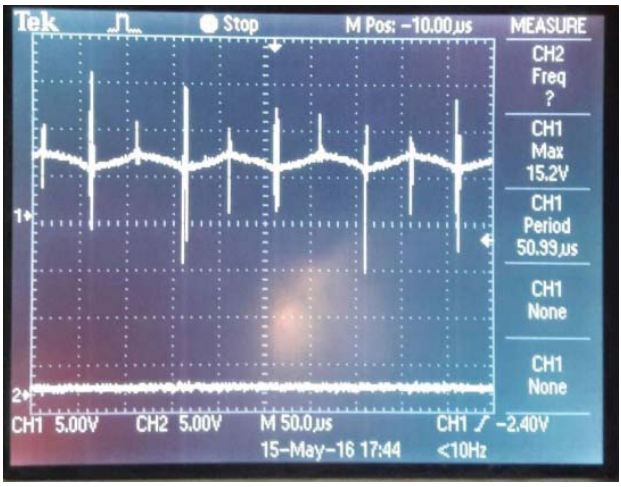

Fig 13. Output voltage waveforms of synchronous buck converter

\section{REFERENCES}

[1] X .Yao, L. Yu, and Y. Luo, "New type non minimum voltage stress of passive lossless soft switch Buck circuit design," Journal of instruments and meters, vol. 32, no. 3, pp: 667-672, 2011.

[2] G. Meng, S. Qu, and H. Cai, "Based on the discrete variable structure control of DC-DC converter," Control theory and applications, vol. 20, no.1, pp: 63-65, 2003.

[3] S. Tan, Y. Lai, M. Cheung, "On the practical Design of a sliding Mode Voltage Controlled Buck Converters," IEEE Transaction Power Electronics, vol. 20, no. 2, pp: 425-431, 2005.

[4] S. Choih, Y. Pair,Y. Cho, "Global sliding mode control Buck,” IEEE Control Systems Magazine, no. 3, pp:21-35, 2003.

[5] Tan, Y. Lai, M. Cheung, "General design issues of sliding mode controllers in DC-DC converters," IEEE Industrial Electronics, no. 5, pp: $1160-1172$.

[6] J. Li, F. Wang, L. Li, "Research and implementation of sliding mode control of Buck converter," Power technology, vol. 134, no. 2, pp: 190-191, 2010

[7] Y. Zheng. "Digital control and its characteristics of DC - DC converter,"Journal of electronic measurement and instrument, vol. 13, no. 3, pp: 44-50, 1999

[8] L. Feng, “The study of the digital PID control algorithm,"Journal of Liaoning University, vol. 32, no. 4, pp: 367-370, 2005.

[9] J. Guan, "New digital control method of DC - DC converter research and contrast," Electronic measurement technology, vol. 4, no. 3, pp: 36-39, 2013.

[10] S. Chuodhury, "Designing a TMS320F280x Based Digital Controlled DC-DC Switching Power Supply," TI Digital Power, C2000 DSP and System Power Management, July 2005. 